Cinémas

Revue d'études cinématographiques

Journal of Film Studies

STAM, Robert. Subversive Pleasures. Bakhtine, Cultural

Criticism and Film. Baltimore and London : The Johns Hopkins

University Press, 1989, 274 p.

\title{
Michèle Garneau
}

Volume 2, numéro 1, automne 1991

URI : https://id.erudit.org/iderudit/1001059ar

DOI : https://doi.org/10.7202/1001059ar

Aller au sommaire du numéro

Éditeur(s)

Cinémas

ISSN

1181-6945 (imprimé)

1705-6500 (numérique)

Découvrir la revue

Citer ce compte rendu

Garneau, M. (1991). Compte rendu de [STAM, Robert. Subversive Pleasures.

Bakhtine, Cultural Criticism and Film. Baltimore and London : The Johns

Hopkins University Press, 1989, 274 p.] Cinémas, 2(1), 177-186.

https://doi.org/10.7202/1001059ar d'utilisation que vous pouvez consulter en ligne.

https://apropos.erudit.org/fr/usagers/politique-dutilisation/ 
STAM, Robert. Subversive Pleasures. Bakhtine, Cultural Criticism and Film. Baltimore and London: The Johns Hopkins University Press, 1989, 274 p.

Si les ouvrages de Mikhaïl Bakhtine, et tout particulièrement les contre-propositions polémiques contenues dans Marxisme et philosophie du langage ${ }^{1}$ envers le Cours de linguistique générale avaient été traduits plus tôt en France, c'est-à-dire avant la décennie soixante-dix, l'impact du structuralisme en sciences humaines eût peut-être été tout autre. On sait que les conditions d'implantation institutionnelle des Études cinématographiques dans les années soixante et soixante-dix sont inséparables de la discipline à laquelle on assignait alors un rôle de compréhension prédominante par rapport à toutes les autres. Le structuralisme a été l'écran théorique le plus visible et le plus lumineux de cette période et c'est à travers lui que se sont projetées un grand nombre de disciplines. Pour se donner une légitimité académique et des garanties de rationalité, les Études cinématographiques ont trouvé auprès de la linguistique saussurienne et du structuralisme un dispositif cognitif et des outils méthodologiques qui lui ont permis de se constituer en objet spécifique. Que se serait-il passé si on avait accordé aux propositions théoriques de Bahktine et à sa translinguistique toute l'importance qu'on s'entend aujourd'hui de plus en plus à lui reconnaître? C'est par la réponse à cette question et par la proposition d'une translinguistique du film que commence Robert Stam dans son chapitre historico-théorique intitulé Translinguistics and Semiotics:

A translinguistic approach to a theory of film language would allow us, after having "discovered" cinema specificity, to reappreciate the links of film to other "series" or semiotic systems, to reaffirm the affinities of film studies to other disciplines, and to reenvision the relation between film history and the larger historical trajectory of narrative and discursive forms (p. 56).

La translinguistique sert de base à Robert Stam pour une approche socio-sémiotique du cinéma et des mass média. "My purpose here will not be to develop a full-blown «Bakhtinian Translinguistics of the Cinema», but rather to sketch out some of the directions such a project might take" (p. 39). Bakhtine appelle cette science trans ou meta linguistique parce qu'elle se consacre à des problèmes qui sortent du cadre de la linguistique, sans pour autant exclure certains des postulats de celle-ci. La translinguistique a pour objet l'énoncé qui a deux aspects: un qui lui vient de la langue, et l'autre du contexte d'énonciation. 
L'énoncé possède à la fois une signification linguistique, c'est-àdire réitérable et virtuelle, et une signification trans ou meta linguistique, c'est-à-dire non réitérable et concrète. Le contexte d'énonciation, le rapport à un locuteur ou à un spectateur constituent ce que Bakhtine appelle la socialité de l'énoncé.

$\mathrm{Ce}$ que Bakhtine propose c'est une science capable d'incorporer la diachronie et une théorie de la signification. Il s'agit de maintenir une tension dialectique entre le fonctionnement interne du texte, c'est-à-dire sa structure, et ses déterminations spatio-temporelles, à savoir sa socialité, de ne pas hypostasier les lois internes du système ni de se satisfaire d'approches sociologiques externes.

Considérer le film comme un énoncé consistera donc à ne pas l'isoler de son contexte de production ni de ses contextes de réception. Le texte n'est pas une entité autotélique et autosuffisante comme chez les formalistes, mais un lieu dynamique, changeant, dans lequel s'effectuent des échanges dialogiques. Il passe d'un locuteur à un autre, d'un contexte à un autre, d'une collectivité sociale à une autre. Dans une approche translinguistique il faut tenir compte des sujets et de la façon dont ils redistribuent le système de signe. C'est par cette mobilité du signe que la translinguistique peut articuler la question du spectateur et critiquer le mythe de l'unique spectateur. "The textually constructed reader/spectator does not necessarly coïncide with the sociohistorical reader/spectator" (p. 43).

Dans le contexte des années vingt, Bakhtine s'élevait contre deux idéologies linguistiques tenues pour idéalistes: l'objectivisme abstrait de la synchronie saussurienne et le subjectivisme individualiste des études stylistiques allemandes (Vossler, Spitzer). Il s'agissait de dépasser tout le champ sociologisant issu du XIX ${ }^{\mathrm{e}}$ siècle et primitivement marxisant et de faire front au structuralisme immanentiste des formalistes. Dans le compte rendu qu'il fait des grandes prises de position de Bakhtine, Stam a tendance, comme d'ailleurs l'ont fait de nombreux commentateurs avant lui, à durcir les oppositions. Dans la société des lendemains de la révolution, il y avait des raisons politiques à la clôture du texte des formalistes. En face du champ littéraire sociologisant qui se constituait, ils furent contraints d'outrer leurs positions. Il y a dans le travail des formalistes les bases d'une approche socio-historique de la littérature, une tentative - et une des premières - de théorisation des séries. Stam est fidèle ici au grand geste argumentatif marxiste: mettre côte à côte deux propositions erronées mais dominantes et interposer un troisième terme non encore aperçu. 
Le même réductionnisme est à l'œuvre à l'égard de Saussure. Stam réduit et ramène la postérité du Cours de linguistique générale au structuralisme français des années soixante. On sait aujourd'hui qu'il y avait dans le Cours de linguistique générale les bases d'une sémiologie non immanentiste. Que Bakhtine n'ait pas perçu ce potentiel n'est pas une raison pour continuer à l'ignorer, d'autant plus qu'un grand nombre de sémiologies se sont développées à partir de lui. Il n'y a qu'à penser au linguiste marxiste saussurien Luis Prieto, au praguois Jan Mukarovsky, qui très tôt ont fait un usage historico-critique des concepts saussuriens ${ }^{2}$.

Il s'agit donc, à travers les prises de position de Bakhtine, de défendre un point de vue idéologique, axiologique et intersubjectif dans l'approche du texte filmique, et de le défendre contre tout fétichisme du code, de l'objectivisme, de l'ahistoricisme et du logicisme binaire. En fait, la théorie cinématographique aurait tout à gagner à articuler la translinguistique de Mikhaïl Bakhtine à la sémiologie de Christian Metz.

Just as Bakhtin "needed" Saussure and the formalist in order to "go beyond" them, so a cinematic translinguistics needed Metz in order to transcend his word. (...) Metz must be credited, to paraphrase Bakhtin's tribute to the formalist contribution to literary study, with "isolating the (cinematic) text as such, revealing its structure, determining the forms and variations of this structure, defining elements and its functions. But if it is not to fall into ahistoricism and scientism, the Metzian model also "needs" Bakhtin (p. 55-56). ${ }^{3}$

Metz aiderait là où Stam reste imprécis par rapport à la conceptualisation de Bakhtine. En effet, si l'énoncé tel que le définit Bakhtine peut être aussi bien un mot qu'une œuvre tout entière, comment et selon quels critères le définir pour l'analyse filmique? L'énoncé a t-il des prédicats définitoires ou est-il une décision de l'analyste? Dans les nombreuses analyses qu'il amorce et dans celle plus poussée qu'il fait du film de Zelig, Stam ne semble pas préoccupé par cette question de méthode. C'est davantage par la constitution d'un corpus le plus vaste possible et par une extrapolation des concepts que par un travail d'acclimatation de ceux-ci que Stam cherche à démontrer la rentabilité de l'heuristique bakhtinienne au cinéma.

Une fois posée la translinguistique et amorcé le passage théorique et généralisant de l'heuristique bakhtinienne au cinéma, Stam propose d'appliquer d'autres concepts pour l'aspect plus analytique de sa démonstration. Les catégories qu'il travaille sont le dialogisme, l'hétéroglossie, la polyphonie et le carnaval. L'auteur cherche à démontrer le potentiel d'opérativité de ces 
concepts au cinéma. La déshistorisation et la désynchronisation dans l'application que subissent ces concepts ne leur enlèvent nullement de leur force opératoire. Il y a une efficace transhistorique dans les concepts bakhtiniens sur laquelle tout le monde s'entend. En parlant du carnaval Stam écrit: "The perennial tropes of carnival" (p. 173). «Il y a dans le carnaval écrit André Belleau, un principe transhistorique actif procédant des profondeurs de la vie quotidienne et du peuple» 4 . Ce principe transhistorique actif ne doit cependant pas être confondu avec la carnavalisation littéraire. Il y a le carnaval en tant que rituels de comportement dans la société hors texte et la carnavalisation en tant que phénomène textuel. La carnavalisation, très généralement, désigne l'inscription textuelle de la culture populaire. Bakhtine a proposé cette notion dans son Rabelais $^{5}$, notion intimement liée à une époque spécifique de l'histoire littéraire - la Renaissance - et à la naissance du roman moderne. Le fait de supposer, comme le fait Robert Stam, une carnavalisation dans des textes filmiques contemporains nécessite donc que l'on n'en fasse pas uniquement une question de contenus sémantiques. $\mathrm{Si}$ on admet que les concepts de Bakhtine ont une valeur transhistorique et qu'on propose d'élaberer à partir d'eux une typologie propre à faire de l'analyse filmique, on doit essayer de saisir le phénomène de textualisation qu'est la carnavalisation, moins en terme de représentation ou de contenus sémantiques qu'en terme de structuration. Stam s'en tient trop souvent aux contenus sémantiques. Or les contenus sémantiques des carnavals sont extrêmement variés. On peut en accumuler toute une série et ainsi ouvrir très largement des séries filmiques correspondantes. L'ouvrage de Stam souffre de cette pléthore. Mentionnons comme caractéristiques carnavalesques principales l'exigence vitale et universelle de participation, la suppression joyeuse des distances entre les hommes, l'expression concrète des expressions refoulées, l'inconvenance parodique et profanatrice 6 . Ces caractéristiques ne sauraient être confondues avec les critères de validité textuelle du concept de carnavalisation. C'est précisément là que réside la confusion chez Stam. Dans les films-textes qu'il retient comme carnavalesques il ne s'agit souvent que de phénomènes locaux, discontinus. Stam analyse des films de Fellini, Pasolini, Wertmuller, Immamura, Buñuel, Godard et veut considérer les films de ces auteurs comme faisant partie d'une tradition carnavalesque plutôt que comme des films qui déconstruisent les conventions du cinéma dominant:

But if we are to see such filmmakers as Bunuel, Godard, Ruiz, and others not as the mere negation of the dominant tradition but 
rather as perpetuators of another tradition, our perspective must be quite different. We would see them as the renovators of a perennial mode whose protean vitality derives not only from individual invention but also from renewed contact with an often repressed but ultimately irrepressible generic tradition (p. 10).

L'indifférenciation entre le carnavalesque et la carnavalisation, qui est une conséquence de l'extrapolation que fait subir Stam aux concepts de Bahktine a des conséquences fâcheuses quant à la rentabilité des ces concepts. Si extrapoler signifie appliquer une chose connue à un autre domaine pour en déduire des hypothèses et des conséquences, je dirais que les hypothèses d'applicabilité de l'heuristique bakhtinienne au cinéma sont très convaincantes mais que leurs conséquences analytiques laissent un peu à désirer, et cela par un éparpillement trop grand du corpus et par une extrapolation trop grande des concepts. Toute la difficulté est dans le passage de la constatation des caractères du carnaval à l'analyse textuelle. Comment fonder ce passage du point de vue théorique et en garantir la sûreté et la rentabilité du point de vue méthodologique? Stam y parvient dans son chapitre "Of Cannibals and Carnivals" consacré aux productions culturelles du Brésil. L'auteur rapproche ici les stratégies de l'anthropophagie brésilienne à celles du carnaval bakhtinien. Le concept-figure «anthropophagie» fut lancé en 1928 au Brésil par Oswald de Andrade dans un manifeste avant-gardiste et fut repris dans les années cinquante par Haroldo de Campos qui en fit un concept apte à rendre compte de la spécificité de la littérature latino-américaine, et brésilienne en particulier. Dans le contexte postcolonial et tiers-mondiste du Brésil, l'anthropophagie signifie l'appropriation productrice de la culture autre.

"Anthropophagy" in this sense, can be seen as another name for Kristeva's "intertextuality" or Bakhtin's "dialogism" and "carnavalisation", but this time in a context of neocolonial cultural domination (p. 125).

Des chanchadas, genre le plus populaire au Brésil, au cinéma nuovo, l'auteur montre comment les stratégies carnavalesques et cannibalesques servent à se prémunir, entre autres, de l'envahissement du cinéma américain. Liberté d'intrigue, fusion du fantastique et du naturalisme, contraste et oxymoron, éléments d'utopies, diversité des voix idéologiques, moquerie des voix officielles, etc. sont les modalités principales de ces genres. La parodie est le grand procédé de la carnavalisation et de l'anthropophagie. Il y a toujours, dans la parodie, une double voix, la réutilisation d'un discours autre. 
This double-voicedness is implicit in the modernist trope of anthropophagy, of devouring the foreign, the alien word, in order to affirm the national (p. 155).

Stam montre bien cependant que la carnavalisation, comme l'anthropophagie, ne peuvent être réduites à une simple opération de renversement qui affirme les valeurs de l'altérité ou du pouvoir. La pratique du cinéma brésilien est marquée par la manipulation ludique, euphorisante- et criticue de divers matériaux discursivo-cinématographiques. Les stratégies textuelles de ces deux figures sont infiniment complexes et ont un pouvoir de déstabilisation. En associant la carnavalisation à l'anthropophagie, et en rapprochant leurs stratégies discursives, Stam réinvestit le concept de Bakhtine d'une logique stratégique et d'un sens historique qui lui redonne toute sa force opératoire. Cette reterritorialisation du concept de Bakhtine sur l'analyse de la situation sociale et culturelle brésilienne est extrêmement judicieuse et éclairante.

C'est principalement à travers l'analyse du film Zelig de Woody Allen que Stam fait ressortir les possibilités dialogiques du cinéma. Tout un chapitre est consacré à Stardust Memories et Zelig. Stam fait la démonstration que "Zelig not only thematizes chameleonism through its protagonist's «uncanny» ability to take on the accent, profession, and ethnicity of his interlocutors, but it also practices chameleonism on a discursive level" (p. 200) et que "the self needs the collaboration of others in order to author and define itself, just as the film-text Zelig literally derives its existence from preexisting texts" (p. 218). Le dialogisme chez Bakhtine consiste dans la relation des divers discours qui modèlent l'énonciation romanesque. La circulation des langages est le phénomène caractéristique de la polyphonie. Dans Zelig, Stam montre comment les genres cinématographiques et les types d'images créent le dialogisme, comment ils s'opposent, s'appellent, se répondent, s'hybrident et s'encadrent les uns les autres. Toute la théorisation du mot et du discours d'autrui développée dans La Poétique de Dostö̈evski ${ }^{7}$ et dont le potentiel n'a pas encore été exploité pour le texte littéraire et encore moins pour le texte cinématographique est ici finement éprouvée sur ces deux films de Woody Allen.

Après avoir considéré le film comme langage, Stam consacre un chapitre sur le langage dans les films en se penchant tout particulièrement sur les aberrations et les courts-circuitages identitaires qu'entraînent le doublage et la postsynchronisation dans un pays du tiers monde comme le Brésil. Un exemple de cela est le cas où les voix sont des voix brésiliennes mais où les images et la 
mentalité sont américaines. La perspective politique de ce problème et ses conséquences culturelles ont été très peu étudiées jusqu'ici. On part donc du principe que les échanges linguistiques sont aussi des rapports de pouvoir symboliques. "Languages can serve not only to oppress and alienate but also to liberate (...) It's is always susceptible to subversion" (p. 84). Le concept bakhtinien d'hétéroglossie servira de base à cet examen du cinéma en tant que pratique discursive révélatrice de relations de pouvoir. L'hétéroglossie consiste dans l'interaction de plusieurs langages générés par les différences sexuelles, raciales, économiques, etc.

The proliferation of competing and intersecting social "languages" cohabitation within a "single" language. We are referring, then, not only to the clearly distinct idioms (English, French, Russian, Arabic) recognized as linguistic unities by grammars and lexicons, but also to the diverse class, generational, and professional languages that coexist within a single culture or speech community (p. 59).

Dans ce chapitre intitulé Language, Difference, and Power, Stam soulève une série de questions très intéressantes concernant des analyses hétéroglossiques possibles. "What is the linguistic dimension of an emerging cinema within a situation of «unstable bilingualism» such as that of Quebec?" (p. 78). Il s'agit de cas où "possession of two languages is not here a matter of having two tools but rather entails participation in two conflicting psychic and cultural realms" (p. 80). Il s'agirait d'examiner dans ce type d'analyse comment on essaie dans certains films de conjurer un pouvoir linguistique, l'impérialisme de la langue américaine par exemple. Il ne suffit pas que l'américain soit la plus grande langue véhiculaire, d'après des circuits économiques et financiers; il se charge aussi, par le cinéma, de fonctions référentielles et mythiques. On voit comment les positions translinguistiques de Bakhtine relativisent le caractère informatif du langage et l'assimilation de la langue avec un code pour subordonner les problèmes linguistiques à une véritable pragmatique ou politique. L'analyse hétéroglossique permet de dégager les agencements de pouvoir mis en jeu dans une langue, les possibilités linguistiques de lutte contre ces pouvoirs. Etudier les fonctions incarnables dans une langue c'est tenir compte directement des facteurs sociaux, des rapports de forces, des centres de pouvoir très divers; on essaie d'évaluer le système hiérarchique et impératif du langage comme exercice de pouvoir ou résistance à cet exercice. Ce type d'analyse du langage dans les cinématographies des minorités reste à faire. 
Fidèle à son corpus carnavalesque, Stam analyse aussi, dans ce chapitre, des films de Buñuel - comment par exemple L'Ange exterminateur constitue une déconstruction comique du genre de discours «conversation de salon» -, des films de Godard, Sembene, Rocha et Ruiz.

Dans les chapitres consacrés à la pornographie The grotesque body and Cinematic Eroticism et aux mass média Envoi: Bakhtin and Mass-Media critique Bakhtine me semble un peu à la remorque de l'argumentation de Stam qui voudrait voir ces pratiques rehaussées par l'heuristique bakhtinienne.

Stam essaie de démontrer comment les catégories de Bakhtine pourraient contribuer à sortir le discours critique sur la porno et sur les mass média de considérations moralisantes. Il ne s'agit pas d'éliminer ces pratiques, prévient-il, mais de les rendre carnavalesques et polyphoniques. Si le machisme, le patriarcat, l'hétérosexualité et le puritanisme dominent le marché de la porno, ce n'est pas une raison, selon Stam, pour les éliminer. "The problem is not that porn is vulgar but that it is monologic"(p. 167). Le sexe dans la porno est autosuffisant, hypostasié. Or les langages sexuels sont multiples; il y a une hétéroglossie de l'érotisme et il faudrait pouvoir mettre en image cette hétéroglossie. Stam est pour une "translinguistics of eroticism", "a carnal polyphony", "a sexual heteroglossy". Un modèle bakhtinien de la sexualité nous sortirait de ces images monologiques.

Dans son chapitre sur les mass média, Stam critique l'incapacité de la critique de gauche à faire face aux mass média et à les traiter comme des réalités constitutives de l'époque. "How might Bakhtin help us transcend some of the sterile analytical dichotomies of the traditional left?" (p. 25). Cette critique commettrait trop souvent la méprise catégoriale d'aborder ces phénomènes moralement en s'indignant ou en s'enthousiasment face à l'objet et en proposant des solutions de rechange dichotomiques stériles. Plutôt que de dénoncer les mass média comme une pratique rétrograde, on doit au contraire chercher à comprendre comment cette expression collective d'affects et de désirs réels fonctionne et pourquoi elle fonctionne. La polyphonie pourrait permettre un traitement dialectique affiné pour une analyse de la politique culturelle des mass média.

Stam parle du simulacre carnavalesque des mass média, de ses formes dégradées de carnavalisation, de son faux grand dialogue, de l'utopie mass-médiatique, et des "pseudo polyphonic discourse": 
A Bakhtinian analysis would be aware of the dangers of "pseudopolyphonic" discourse, one that marginalizes and disempowers certain voices, and then "dialogues" with a puppetlike entity that has already been forced to make crucial compromises (p. 232).

The American mass media are fond of weak or truncated forms of carnival that capitalize on the frustrated desire for a truly egalitarian society by serving up distorted version's of carnival's utopian promise... (p. 226).

C'est comme si peu à peu Stam abandonnait la fonction théorico-analytique des concepts de Bakhtine pour un usage prescriptif-humaniste. À l'égard de la pratique cinématographique pornographique et des mass média, il $\mathrm{y}$ a un phénomène de condamnation en faveur de l'outil cognitif qui pourrait relever ces pratiques.

How can Bakhtin aid in the elaboration of a radical hermeneutic that goes beyond dogmatism, beyond puritanism, beyond economism, and beyond cultural defeatism? How might Bakhtinian conceptualizations help us analyze, teach and finally generate mass-mediated culture? Why, in short, do we "need" Bakhtin? (p. 25).

Une question ici s'impose concernant le recours à Bakhtine: peut-on appliquer une conceptualité historique moderne à une pratique qui se développe finalement à l'encontre de cette conceptualité? Dans le cas des mass média, ne faudrait-il pas développer de nouveaux outils d'analyse? Certes nous avons besoin de Bakhtine, mais jusqu'à quel point sa théorisation peut-elle nous permettre de ne pas rater la spécificité historique des mass média?

Parce qu'elles proviennent d'une théorie culturelle qui traverse en les intégrant plusieurs disciplines (linguistique, sociologie, histoire et littérature) et parce qu'elles ne constituent pas une théorie totalisante et homogénéisante, les propositions théoriques de Mikhaill Bakhtine pourraient permettre de nouveaux «décollements» en théorie et en analyse cinématographiques. Elles pourraient aussi protéger, ce qui n'est pas peu dire, des écueils du syncrétisme dont a déjà beaucoup trop souffert la théorie du cinéma. Le travail de Stam est un travail de pionnier et constitue ce que l'on pourrait appeler les prolégomènes indispensables à toute application future de Mikhaïl Bakhtine. 


\section{NOTES}

1 Mikhail Bakhtine (V.N. Volochinov), Le Marxisme et la philosophie du langage, essai d'application de la méthode sociologique en linguistique (Paris: Minuit, 1977).

2 Sur cette question Marc Angenot a écrit deux articles: «Bakhtine, sa critique de Saussure et la recherche contemporaine», Études françaises 20/1 (printemps 1984) p. 7-19, et "Institutional Distorsions of Saussure: Structuralism as Syncretism", John Fekete (dir.), The Structural Allegory (Minnesota University Press, 1983).

3 Robert Stam aborde plus en détail cette question dans un texte intitulé "Film and language: From Metz to Bakhtin", R. Barton Palmer (ed.), The Cinematic Text (New York: Ams Press, 1989).

4 «Carnavalesque pas mort» publié dans Études françaises, op. cit., p.42.

5 L'Euvre de François Rabelais et la culture populaire au Moyen-Âge et sous la Renaissance (Paris: Gallimard, 1970).

6 Ces caractéristiques sont celles relevées par André Belleau dans «Carnavalisation et roman québécois: mise au point sur l'usage d'un concept de Bakhtine» publié dans Études françaises, $\mathrm{n}^{\circ} 19 / 3$, 1988, p. 54-55-56. Robert Stam les relève aussi en en ajoutant de nombreuses autres.

7 La Poétique de Dostö̈evski (Paris: Seuil, 1970). 